\title{
Recurrent Cholangiocarcinoma in Pregnancy: A Case Report
}

\author{
Antonia P. Francis, MD ${ }^{1}$ Michelle Chang, MD ${ }^{2}$ Cara D. Dolin, MD, MPH ${ }^{4}$ Judith Chervenak, MD, JD ${ }^{1}$ \\ Elyce Cardonick, $\mathrm{MD}^{3}$
}

${ }^{1}$ Department of Obstetrics and Gynecology, New York University Langone Health, New York, New York

Address for correspondence Antonia P. Francis, MD, Department of

2 Department of Medicine, Columbia University Irving Medical Center, Obstetrics and Gynecology, New York University Langone Health, 550

New York, New York

${ }^{3}$ Department of Obstetrics and Gynecology, Cooper University Health

First Avenue, New York, NY 10016 (e-mail: antonia.francis@nyumc.org).

Care, Camden, New Jersey

${ }^{4}$ Department of Obstetrics and Gynecology, University of

Pennsylvania, Philadelphia

Am J Perinatol Rep 2018;8:e261-e263.

\begin{abstract}
Keywords

- cancer

- pregnancy

- cholangiocarcinoma

- hepatobiliary

Hepatobiliary malignancies during pregnancy are extremely rare and portend a poor prognosis. There are only seven published cases of cholangiocarcinoma in an obstetrical patient, all are cases of primary cholangiocarcinoma (1-7). Herein, we describe the first case of recurrent cholangiocarcinoma during pregnancy. The patient did not receive chemotherapy during pregnancy and required prolonged hospitalizations for nutritional and intensive medical support. She delivered preterm, at $30^{2 / 7}$ weeks gestation, after developing pre-eclampsia with severe features. The infant was healthy, with no malformations, and currently exhibits no neurological or behavioral sequelae at 8 months of age. We discuss themanagement considerations inherent to this complex clinical scenario including metastatic disease severity, ethical considerations, and palliative care treatment options.
\end{abstract}

Hepatobiliary malignancies during pregnancy are extremely rare and portend a poor prognosis. There are only seven published cases of cholangiocarcinoma in an obstetrical patient, all are cases of primary cholangiocarcinoma. ${ }^{1-7}$ There is limited data regarding the recurrence of cholangiocarcinoma in pregnancy and pregnancy outcomes in the presence of recurrent disease. We discuss the management considerations inherent to this complex clinical scenario including metastatic disease severity, ethical considerations, and palliative care treatment options.

\section{Case Report}

A 36-year-old gravida 3 para 2 at $23^{0 / 7}$ weeks gestation with a history of stage II $(\mathrm{T} 2 \mathrm{~N} \times \mathrm{M} 0)$ cholangiocarcinoma presented with painless jaundice, emesis, and transaminitis concerning for an obstructive pathology. The pregnancy had been uncomplicated prior to this presentation. The

received

February 22, 2018

accepted

September 5, 2018
DOI https://doi.org/

10.1055/s-0038-1675376. ISSN 2157-6998. patient was diagnosed with cholangiocarcinoma 14 months prior to presentation after undergoing elective laparoscopic cholecystectomy for cholelithiasis. One month later she underwent exploratory laparotomy and partial hepatectomy with negative nodal involvement. She received adjuvant gemcitabine and radiation therapy but never completed the full course as instructed. Five weeks prior to conception, surveillance imaging was negative. She discovered her pregnancy at 7 weeks of gestation.

Given the patient's history, recurrence of cholangiocarcinoma was strongly suspected on presentation. Magnetic resonance cholangiopancreatography showed severe biliary ductal dilatation and a poorly defined common bile duct lesion. Magnetic resonance imaging of the abdomen revealed large bilateral multiloculated adnexal masses, concerning for Krukenberg's tumors. The patient initially underwent interventional radiology-guided transhepatic biliary catheter placement, as well as a failed biopsy attempt due to tissue
Copyright $\odot 2018$ by Thieme Medical Publishers, Inc., 333 Seventh Avenue, New York, NY 10001, USA. Tel: +1(212) 584-4662.
License terms

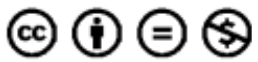


fragility. After this procedure, her symptoms improved and she was discharged home in good condition. She was readmitted 6 days later for symptoms of nausea, large volume emesis, fatigue, and anorexia. A computerized tomography scan of the abdomen and pelvis showed no evidence of obstruction, but due to worsening transaminitis, acute kidney injury, ${ }^{8}$ and lactate elevation, the patient was transferred to the medical intensive care unit for stabilization and received antenatal corticosteroids. Her persistent emesis required total parenteral nutrition and exploratory endoscopy with placement of a jejunostomy tube. On hospital day 14 , endoscopy revealed a partial duodenal obstruction with a small patent area and inconclusive duodenal biopsies (also due to tissue fragility). On hospital day 21 , repeat biopsy results revealed moderately differentiated invasive adenocarcinoma with tumor cells diffusely positive for CK7 and CK19 and focally positive for CK20, features consistent with recurrent cholangiocarcinoma. A palliative duodenal stent was placed. Serial multidisciplinary meetings were held throughout her hospital course to coordinate close surveillance of maternal and fetal status and maintain an active dialogue regarding delivery planning and possible therapeutic interventions. In addition, the care team frequently discussed with the patient and her family, her wishes regarding her desired pregnancy and day-to-day care. The patient was offered palliative chemotherapy but there were concerns of her becoming refractory to treatment. She understood that chemotherapy was only palliative and unlikely to significantly extend her life and wished to spare her fetus any exposure, thus she elected to delay palliative chemotherapy until postpartum. On hospital day 24 (27 weeks of gestation), the patient tolerated a regular diet and was discharged home with close outpatient follow-up.

At $29^{4 / 7}$ weeks gestation, she represented with malaise, hypovolemia, and acute kidney injury, as evidenced by elevated creatinine and anuria. During this admission, she subsequently developed pre-eclampsia with severe features and worsening, uncontrolled pain due to her metastatic disease burden. She received rescue dose corticosteroids and the decision was made to induce labor at $30^{3 / 7}$ weeks of gestation due to severe hypertension uncontrolled on medications. A nonanomalous, viable male infant was delivered vaginally with a birth weight of 1,620 grams (65\%) and Apgar's of 9/9. The infant had an uncomplicated neonatal intensive care unit course, requiring only noninvasive ventilation. Head ultrasound was normal and the infant was discharged after a 36-day hospital stay. He is doing well at 8 months with no apparent sequelae. Placental pathology was negative for evidence of malignancy.

The patient was transferred to the medicine unit on postpartum day 4 for continued palliative care. The patient was unable to spend significant time in the neonatal intensive care unit due to deteriorating state but continued to play an active role in health decisions for her infant through multidisciplinary meetings with the neonatal team, social work, and her proxy. On postpartum day 17 , she received a venting percutaneous endoscopic gastrostomy tube and was discharged home on postpartum day 19 . The patient died at home 1 month from final discharge.

\section{Discussion}

In a pregnant patient, cholangiocarcinoma poses significant management challenges given the rarity and poor prognosis of the condition. Maintaining nutritional status, providing continuous palliative care, and balancing ethical concerns for the mother-baby dyad all contributed in making this case particularly complex.

Malignancy is rare in pregnancy, occurring in 1 in 1,000 to 1 in 6000 pregnancies. $^{9-11}$ The most frequently occurring cancers include breast, cervical, hematologic, thyroid, colon, ovarian, and melanoma. ${ }^{9}$ Provided that pregnant patients with cancer are similarly treated, survival does not differ from the nonpregnant population. ${ }^{9}$ Cholangiocarcinoma is an uncommon and aggressive malignancy associated with a median survival of 3 to 6 months and an incidence of 0.58 per $100,000 .^{12,13}$ Diagnosis can be challenging, as the typical symptoms of weight loss, malaise, abdominal pain, and night sweats are nonspecific. Often, primary disease is discovered incidentally, as in this case. The mean age at diagnosis is 50 years, the male to female ratio is $1: 1.2-1.5$, and half of individuals with the disease are over 65 , making this 36year-old patient's presentation epidemiologically unusual. ${ }^{14,15}$ While the pathogenesis of cholangiocarcinoma involves cholestasis and chronic inflammation, and several risk factors relating to hepatobiliary inflammation or infection have been identified, most patients (including our patient) do not have a history of any of these conditions. Surgical resection is the only potentially curative treatment, though a majority of patients present with advanced stage disease, and recurrence after resection is common, occurring in up to $64 \%$ of cases. ${ }^{14}$ Given the rarity of cholangiocarcinoma, especially in pregnancy, there is no established time period recommended for completing treatment for the primary cancer and planning a subsequent pregnancy. In general, after treatment for more common cancers occurring during the reproductive years, women are advised to delay pregnancy for at least two disease-free years as this is the time period during which recurrences most likely occur. ${ }^{9}$ This patient never completed the primary treatment recommended then became pregnant 14 months after her initial diagnosis of cholangiocarcinoma.

An optimal management strategy should strive to balance benefit to the mother while decreasing potential harm to the fetus, and should be determined based on extent of disease, recommended course of treatment for nonpregnant women and gestational age at which treatment is considered. Treatment options considered safe in pregnancy include surgery in any trimester and chemotherapy in the second and third trimesters. Chemotherapy is best avoided if possible in the first trimester, during the period of organogenesis. Radiation therapy is associated with teratogenesis, fetal growth restriction, pregnancy loss, intellectual disability, and malignancy in the offspring depending on the gestational age and dosage of exposure. Radiotherapy is 
relatively contraindicated during pregnancy but can be safely administered with modifications if required for specific cancers such as CNS (central nervous system) tumors or aggressive Hodgkin's lymphoma. ${ }^{9}$ The most common complication of malignancy in pregnancy is iatrogenic prematurity but if maternal disease remains stable, delivery at or beyond 37 weeks should be considered. ${ }^{9}$ In the case of recurrent cholangiocarcinoma, chemotherapy could be offered, though it would likely be palliative in nature due to extensive metastasis.

This patient suffered from a large tumor burden and organ dysfunction, ultimately developing severe pre-eclampsia which influenced the team's decision to deliver at $30^{3 / 7}$ weeks of gestation. Until that time, she required an extensive stay in the medical intensive care unit for resuscitative measures including fluid management, close observation of vital signs, and nutritional support and weight gain monitoring. Maternal and fetal both healths are of paramount importance and maintaining the balance between both can be difficult. This complex situation called for continued and frequent communication between all involved providers including maternal-fetal medicine, critical care, oncology, palliative care, nutritional services, neonatology, and social work to maintain an open forum while ensuring optimal patient-centered care.

\section{Conclusion}

This is the first reported case of recurrent cholangiocarcinoma in pregnancy. This is a rare diagnosis and carries a poor prognosis. Frequent, open communication between the patient, multidisciplinary care team, and proxy is essential to establish and meet shared goals. This is especially true when managing a pregnant patient with a terminal illness due to the medical and ethical challenge of balancing maternal and fetal well-being.

\section{Conflict of Interest}

None.

\section{References}

1 Gerli S, Favilli A, Giordano C, Donini A, Di Renzo GC. Mixed hepatocellular carcinoma and cholangiocarcinoma during pregnancy: a case report. Eur J Obstet Gynecol Reprod Biol 2015;187:76-77

2 Sadoon S, Hodgett S. Unusual cause of itching in a pregnancy (cholangiocarcinoma). J Obstet Gynaecol 2008;28(02):230-231

3 Stone S, Girling JC. Deranged liver function tests in pregnancy: the importance of postnatal follow-up. Obstet Med 2009;2(01):32-33

4 Marasinghe JP, Karunananda SA, Angulo P. Cholangiocarcinoma in pregnancy: a case report. J Obstet Gynaecol Res 2008;34(4, Pt 2):635-637

5 Balderston KD, Tewari K, Azizi F, Yu JK. Intrahepatic cholangiocarcinoma masquerading as the HELLP syndrome (hemolysis, elevated liver enzymes, and low platelet count) in pregnancy: case report. Am J Obstet Gynecol 1998;179(3, Pt 1):823-824

6 Devoe LD, Moossa AR, Levin B. Pregnancy complicated by extrahepatic biliary tract carcinoma. A case report. J Reprod Med 1983; 28(02):153-155

7 Purtilo DT, Clark JV, Williams R. Primary hepatic malignancy in pregnant women. Am J Obstet Gynecol 1975;121(01):41-44

8 Khwaja A. KDIGO clinical practice guidelines for acute kidney injury. Nephron Clin Pract 2012;120(04):c179-c184

9 Albright CM, Wenstrom KD. Malignancies in pregnancy. Best Pract Res Clin Obstet Gynaecol 2016;33:2-18

10 Smith LH, Danielsen B, Allen ME, Cress R. Cancer associated with obstetric delivery: results of linkage with the California cancer registry. Am J Obstet Gynecol 2003;189(04):1128-1135

11 Pavlidis NA. Coexistence of pregnancy and malignancy. Oncologist 2002;7(04):279-287

12 Doherty B, Nambudiri VE, Palmer WC. Update on the diagnosis and treatment of cholangiocarcinoma. Curr Gastroenterol Rep 2017;19(01):2

13 Cunningham SC, Choti MA, Bellavance EC, Pawlik TM. Palliation of hepatic tumors. Surg Oncol 2007;16(04):277-291

14 Blechacz B. Cholangiocarcinoma: current knowledge and new developments. Gut Liver 2017;11(01):13-26

15 Tyson GL, El-Serag HB. Risk factors for cholangiocarcinoma. Hepatology 2011;54(01):173-184 